\title{
Enfermedad de Still del adulto: Una gran simuladora. Experiencia clínica basada en $\mathbf{2 0}$ casos
}

\author{
M Ángela Carreño ${ }^{1}$, Aurelio Carvallo ${ }^{1}$, Cecilia Trejo ${ }^{1}$, \\ Francisco Ballestero' ${ }^{2}$, Carola Martínez². \\ Adult Still's disease. \\ Retrospective review of 20 patients
}

Background: Adult Still's disease is an inflammatory disorder characterized by quotidian fevers, and an evanescent rash. Its presentation can be acute or subacute. Aim: To report our experience with Adult Still's disease. Material and methods: Systematic retrospective review of medical records of nine men and 11 women aged between 17 and 57 years, with Still's disease, followed in two public hospitals of Metropolitan Santiago. Results: Eighty percent of patients had a prior different diagnosis. All presented with fever and joint involvement. Eighty percent had malaise, $80 \%$ had odynophagia, $80 \%$ had an evanescent rash, $70 \%$ had myalgias, $50 \%$ had lymph node enlargement and $40 \%$ had splenomegaly. Laboratory showed leukocytosis in $80 \%$ and a high erythrocyte sedimentation rate in all. High ferritin levels were detected in $80 \%$, and became an important diagnosis clue. Initial treatment was based on non steroidal antiinflammatory drugs, however $80 \%$ required steroids and $35 \%$ required methotrexate. Azathioprine, sulphalazine, hydroxychloroquine and leflunomide were used occasionally. Eleven patients had a single episode, nine had a relapsing disease and four had a chronic or persistent mode. Conclusions: Adult Still's disease must be suspected in patients with fever of unknown origin. An early diagnosis and adequate treatment of the disease are associated with a favorable evolution and prognosis (Rev Méd Chile 2009; 137: 1010-6).

(Key words: Antiinflammatory agents, non-steroidal; Fever of unknown origin; Still's disease, adult onset)

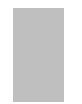

Recibido el 12 de enero, 2009. Aprobado el 25 de junio, 2009.

${ }^{1}$ Servicio de Medicina, Unidad de Reumatología, Hospital San Juan de Dios. ${ }^{2}$ Servicio de Medicina, Unidad de Reumatología, Hospital San Borja Arriarán.

$\mathrm{L}$ a enfermedad de Still del adulto (ESA) es una afección inflamatoria con síntomas articulares y sistémicos característicos, similares a la artritis reumatoidea juvenil de forma sistémica. Descrita

Correspondencia a: Dr. Aurelio Carvallo Valenzuela. Servicio de Medicina, Unidad de Reumatología, Hospital San Juan de Dios. Chacabuco 419, Santiago, Chile. E mail: acarvallo@med.uchile.cl por primera vez por Bywaters en $1967^{1}$ se considera actualmente como causa de síndrome febril de origen inicialmente indeterminado. De etiología desconocida es más frecuente en adultos jóvenes, con prevalencia similar en ambos sexos ${ }^{2-4}$.

Sin síntomas ni alteraciones de laboratorio patognomónicos, requiere de una alta presunción para llegar al diagnóstico, pues simula una variedad de otras enfermedades. Las principales características clínicas son: fiebre en aguja, rash macular o maculo- 
papular evanescente, artralgias o artritis y leucocitosis neutrofílica, acompañadas a menudo de otras manifestaciones como odinofagia, linfoadenopatías generalizadas, hepatoesplenomegalia, alteración de la función hepática, pleuropericarditis y habitualmente ausencia de factor reumatoideo y de anticuerpos antinucleares. Significativa importancia tiene el alza de los niveles de ferritina, útil como elemento diagnóstico, de actividad y de seguimiento de eficacia terapéutica. Diversos autores han propuesto criterios para diagnosticar la enfermedad ${ }^{5,6}$, siendo los más conocidos y sensibles los de Cush $^{7}$ y Yamaguchi ${ }^{8}$.

Por la importancia que esta afección representa, para el médico internista en general y para el reumatólogo en particular, como causa de fiebre de origen desconocido, que es su expresión clínica dominante, consideramos de interés revisar y comunicar nuestra experiencia de los últimos 13 años, analizándola en relación a la internacional.

\section{PACIENTES Y MÉTODO}

En un estudio retrospectivo y descriptivo, se confeccionó un protocolo de estudio y evaluación de pacientes con diagnóstico de ESA controlados entre 1995 y 2008 en las Unidades de Reumatología de los hospitales San Juan de Dios y San Borja Arriarán. Inclusión basada en los criterios diagnósticos definidos por Cush y Yamaguchi (Tabla 1); 20 pacientes cumplieron con los requisitos exigidos. Se analiza edad, sexo, tiempo transcurrido entre inicio de los síntomas y diagnóstico, diagnósticos y tratamientos realizados previos al definitivo, características clínicas (fiebre, compromiso del estado general, compromiso articular, mialgias, odinofagia, rash, linfoadenopatías, esplenomegalia, hepatomegalia, dolor abdominal, pleuritis, pericarditis, neumopatía, alopecia), características de laboratorio (hematocrito, hemoglobina, leucocitos, neutrófilos, plaquetas, velocidad de eritro-sedimentación (VHS), proteína C reactiva (PCR), transaminasas, fosfatasas alcalinas, factor reumatoideo (FR), anticuerpos antinucleares (AAN), ferritina, características radiológicas, tratamiento y evolución. Para exponer los análisis se utilizaron medianas y porcentajes.

\section{Resultados}

Sexo y edad. 9 hombres y 11 mujeres; edad entre 17 y 57 años con mediana de 32 años. Tiempo entre inicio de síntomas y diagnóstico definitivo: 0,6 y 24 meses con mediana de 3 meses. Diagnósticos previos: 16 pacientes (80\%) tuvieron otro diagnóstico previo. Por su diversidad, se ordenaron en 4 grupos: infecciosos, inmunológicos, neoplásicos y neurológicos (Tabla 2).

\section{Tabla 1. C riterios diagnósticos}

\begin{tabular}{|c|c|}
\hline Criterios de Cush & C riterios de Yamaguchi \\
\hline $\begin{array}{ll}\text { Criterios mayores: } \\
\text { - } & \text { Fiebre sobre } 39^{\circ} \mathrm{C} \\
\text { - } & \text { Artralgias/artritis } \\
\text { - } & \text { F reumatoideo }<1: 80 \\
\text { - } & \text { Ac antinucleares }<1: 100 \\
& \\
\text { Criterios menores: } \\
\text { - } \quad \text { Leucocitos }>15.000 / \mathrm{mm}^{3} \\
-\quad \text { Rash evanescente } \\
\text { - } \quad \text { Pleuritis o pericarditis } \\
\text { - } \quad \text { Hepatomegalia-esplenomegalia } \\
\text { - } \quad \text { Lifoadenopatía } \\
\text { Diagnóstico: } 3 \text { mayores } \\
\quad 2 \text { menores }\end{array}$ & $\begin{array}{l}\text { Criterios mayores: } \\
\text { - } \quad \text { Fiebre sobre } 39^{\circ}>1 \text { semana } \\
\text { - } \quad \text { Artralgias/artritis }>2 \text { semanas } \\
\text { - } \quad \text { Rash evanescente } \\
\text { - } \quad \text { Leucocitos }>10.000 \\
\text { - } \quad \text { Neutrófilos }>80 \% \\
\text { Criterios menores: } \\
\text { - } \quad \text { Odinofagia } \\
\text { - } \quad \text { Linfoadenopatía } \\
\text { - } \quad \text { Alza enzimas hepáticas } \\
\text { - } \quad \text { F reumatoideo (-) } \\
\text { - } \quad \text { Ac antinucleares (-) } \\
\text { Diagnóstico: } 5 \text { criterios } \\
\quad 2 \text { deben ser mayores }\end{array}$ \\
\hline
\end{tabular}


Características clínicas (Figura 1). Todos los pacientes presentaron fiebre y 18 de ellos (90\%), desde el inicio de la enfermedad. Compromiso articular (artralgias o artritis) en 100\% en algún momento de la evolución, más frecuente en muñecas y rodillas (Figura 2). El resto de las manifestaciones clínicas más frecuentes fueron compromiso del estado general (80\%), odinofagia (80\%), rash (80\%), mialgias (70\%), linfoadenopatías (50\%) y esplenomegalia (40\%).

Características de laboratorio. En el hemograma, 80\% presentó leucocitosis: sobre 10.000 leucocitos $/ \mathrm{ml}$, y $70 \%$ más de 15.000 leucocitos $/ \mathrm{ml}$; la mitad de los pacientes presentó neutrofilia sobre $80 \%$ y $40 \%$ tuvo hemoglobina menor a 11,5 gr/dl. El 30\% tuvo trombocitosis $(>400.000 / \mathrm{ml})$. La VHS estuvo elevada en 100\%. El 75\% tuvo alteración de las pruebas de función hepática evidenciada por elevación de transaminasas $\mathrm{O}$ fosfatasas alcalinas, desde niveles discretos hasta 6 veces lo normal. En 14 pacientes se evaluó la PCR resultando elevada en 13 de ellos. Se realizó FR en 19 pacientes siendo negativo en 18 $(94,7 \%)$; el positivo lo fue a título bajo (93,6 UI). AAN negativos en $90 \%$. La ferritina sérica medida en 18 pacientes, estuvo elevada en $80 \%$ de los casos, en $55 \%$ de ellos sobre $1.000 \mathrm{mg} / \mathrm{ml}$ y en $5 \%$ sobre $15.000 \mathrm{mg} / \mathrm{ml}$.

Radiología. Se realizó estudio radiológico en 10 pacientes. En 3 se encontró aumento de volumen de partes blandas, con daño articular asociado sólo en 2: uno con disminución de espacio $\mathrm{y}$ erosiones en las radiocarpianas y el otro anquilosis entre carpo y $3^{\circ}$ metacarpofalángica.

Tratamiento inicial. Nueve pacientes inician tratamiento sólo con ácido acetil salicílico (AAS) ( $\geq 3 \mathrm{~g}$ diarios) con buena respuesta en 2; tres con AAS asociado a

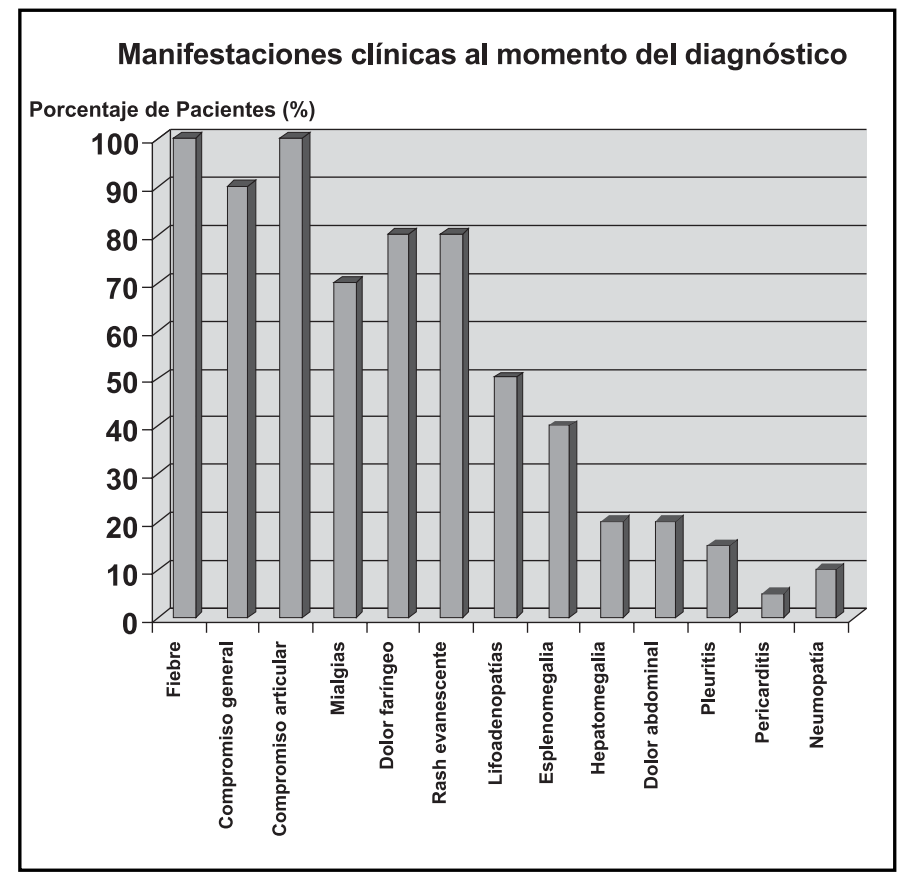

Figura 1.

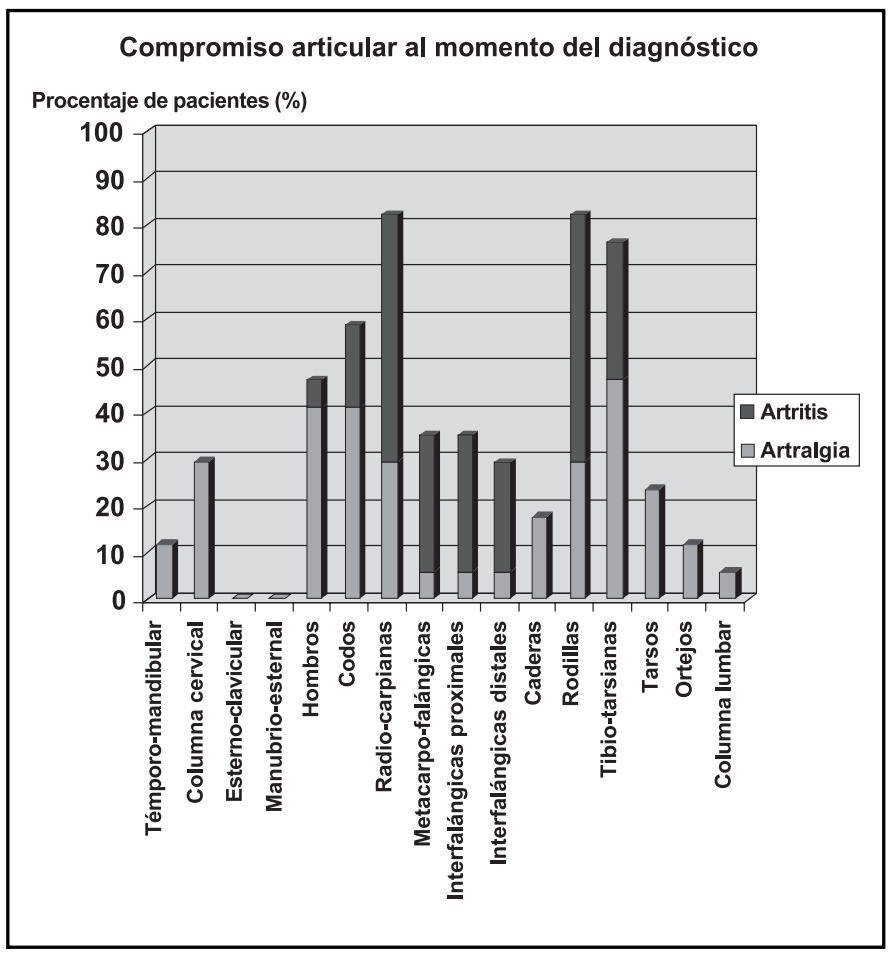

Figura 2. Compromiso articular inicial. 
Tabla 2. D iagnósticos previos al definitivo

1) Infecciosos:

Viral inespecífico

Mononucleosis infecciosa

Faringoamigdalitis aguda

Tuberculosis

Artritis séptica

2) Inmuno-reumatológicos:

Dermatitis alérgica

Artritis reactiva

Artritis reumatoidea

Síndrome de Sjögren

Lupus eritematoso sistémico

Espodiloartritis anquilosante

Vasculitis

Polidermatomiositis

3) Neoplasias:

Leucemia

Linfoma

Neoplasia oculta

4) Neurológicos:

Esclerosis lateral amiotrófica

otro antiinflamatorio no esteroidal (AINE) sin buena respuesta; cuatro iniciaron tratamiento sólo con AINE, con buena respuesta en uno, y 4 pacientes recibieron tratamiento con corticoesteroides desde el inicio, con buena respuesta en 3 de ellos. Hubo 14 pacientes (70\%), sin respuesta al tratamiento inicial, de los cuales sólo 1 había usado corticoesteroides. De los 13 pacientes sin respuesta al tratamiento inicial con AAS o AINEs, en 12 se asoció o reemplazó por corticoesteroides, prednisona o equivalente $(<0,5$ a $1 \mathrm{mg} / \mathrm{Kg} /$ día), con buena respuesta en 5 de ellos, y en uno se asoció metotrexato (MTX) con buena respuesta. De los 8 pacientes que no respondieron a corticoesteroides, se agregó MTX en 6, con buena respuesta en 5, y azatioprina (AZT) en 1, con buena respuesta; un paciente requirió agregar la asociación de metotrexato, sulfazalasina (SULF) e hidroxicloroquina (HQN) y otro de MTX y leflunomida (LFL) para lograr la remisión del cuadro inflamatorio. En resumen, en algún momento de la evolución, dieciséis requirieron corticoesteroides (80\%), doce AAS (60\%), siete AINES (35\%), siete MTX (35\%), uno AZT, uno SULF, uno HQN y uno LFL, usándolas ya sea solas o en combinación.
Evolución de la enfermedad. Once enfermos (55\%) tuvieron evolución monocíclica. De los 9 restantes, cinco (25\%) han sido recidivantes y 4 (20\%) han evolucionado en forma crónica o persistente.

\section{DisCUSIÓN}

En 1897 George F. Still, médico pediatra, describió un tipo de artritis reumatoidea infantil en que al cuadro articular se asociaban fiebre y manifestaciones generales. Se conoce también como artritis reumatoidea juvenil sistémica. En 1971 Bywaters describió un cuadro similar en adultos basándose en 14 pacientes $^{1}$, denominado posteriormente "enfermedad de Still del adulto". Su etiología es desconocida y se han involucrado factores infecciosos y genéticos; por sus características clínicas debe incluirse entre los síndromes febriles de causa inicialmente desconocida. El diagnóstico suele ser difícil, habitualmente por descarte de otras patologías, contribuyendo a que el tiempo previo al diagnóstico definitivo sea frecuentemente prolongado, alcanzando hasta 24 meses en nuestro estudio. La variedad de síntomas dio lugar a diferentes estudios, hipótesis diagnósticas y tratamientos previos, incluido un caso que recibió terapéutica antituberculosa durante un mes. Con este fundamento pensamos que es necesario considerar el diagnóstico de ESA, por simular diversas afecciones, y en especial frente a cuadros febriles de origen no definido.

Como ha sido descrito, encontramos predilección por adultos jóvenes con incidencia semejante en ambos sexos ${ }^{2-4,9,10}$. En el cuadro clínico hay elementos importantes, de alerta y ayuda para diferenciar a la ESA de otros cuadros febriles de origen incierto. Destaca la odinofagia que, como sucedió en nuestra serie, frecuentemente orienta a una farinoamigdalitis y uso de antibióticos sin respuesta. Habitualmente precede en días al cuadro sistémico, pero puede estar incluido en el inicio de éste. Es expresión de una faringitis no infecciosa, no exudativa, que no responde a antibióticos y probablemente manifestación de actividad linfoide acompañada de inflamación. La encontramos en un alto número de pacientes (80\%), lo que concuerda con otras comunicaciones ${ }^{4,9}$. Puede preceder o ser también parte de episodios de recidivas. 
El compromiso general y disminución de peso pueden orientar a etiologías infecciosas o neoplásicas 9 , más aún si se agrega fiebre, presente en todos nuestros casos, que suele ser precedida de calofríos. Habitualmente elevada, superando los $39^{\circ} \mathrm{C}$, tiende a presentarse a la misma hora del día, frecuentemente hacia la tarde y noche, aunque en ocasiones es matinal ${ }^{3,9}$

El rash, presente en $80 \%$ de nuestros enfermos, destaca por ser fugaz o evanescente; de color "asalmonado", macular o maculopapular, puede acompañarse de prurito y dermografismo localizándose preferentemente en el tronco y zona proximal de extremidades. Habitualmente asociado a alzas febriles, es a veces tan sutil que pasa desapercibido para paciente y médico ${ }^{2,3,9}$, de tal modo que si se piensa en el diagnóstico, debe buscarse en forma dirigida, constituyendo un elemento clave. Fiebre y rash hacia el final del día les ha valido la denominación de "síntomas del residente".

Siendo el compromiso articular un elemento importante y muy frecuente (todos nuestros casos), suele ser inicialmente poco expresivo, siendo enmascarado por los síntomas generales, dificultando el diagnóstico. El examen acucioso evidencia alteraciones articulares no mencionadas inicialmente por el enfermo, más afectado por su compromiso general. Incluso 5\% de los pacientes nunca presentan artritis, o bien, ésta puede ser sólo transitoria ${ }^{11}$. De inicio óligo o mono articular, puede progresar hacia poliarticular o bien, presentarse de esta última forma desde el comien$\mathrm{zO}^{2,9}$. Encontramos mayor compromiso de radiocarpianas, rodillas y tibiotarsianas, lo que concuerda con la experiencia extranjera $3,9,11$. La columna cervical, afectada en un tercio de nuestros casos, cuando estuvo presente, constituyó un elemento clínico destacado.

La mitad de nuestros enfermos presentó linfoadenopatías, especialmente cervicales, que agregadas al compromiso general y fiebre, pueden inducir al diagnóstico de un cuadro linfoproliferativo. Frente a la duda, la biopsia e histología de linfoadenitis inespecífica, permite descartar una enfermedad maligna ${ }^{12}$.

No hay exámenes de laboratorio específicos de la afección, pero sí parámetros que en su conjunto orientan al diagnóstico. La VHS y la PCR están invariablemente elevadas, muchas veces en forma significativa; sobre la mitad de nuestros enfermos tuvieron una VHS sobre 100 mm, y más de dos tercios una PCR mayor de $100 \mathrm{mg} / \mathrm{dl}$. Si bien los cambios de la serie roja y plaquetaria son inespecíficos y propios de un proceso inflamatorio prolongado (tendencia a la anemia normocítica normocrómica y trombocitosis), la serie blanca destaca por la presencia de leucocitosis y neutrofilia $3,9-11$, en 90\% y 80\%, respectivamente en nuestra serie, que por su relevancia constituyen criterios diagnósticos.

La ferritina plasmática ha adquirido gran importancia en la ESA como orientador diagnóstico y de actividad, postulándose su inclusión como criterio diagnóstico $^{13}$. Su alza es expresión de la respuesta inflamatoria, y su aumento en la ESA es probablemente por desvío de fierro lábil hacia los depósitos de ferritina ${ }^{6}$, o bien, por liberación de ferritina de los hepatocitos dañados 9 . Estudios moleculares demuestran que la ferritina se glicosila en el plasma y normalmente el porcentaje de glicosilación es entre 50\% y 80\%, el que varía durante estados de inflamación aguda y crónica. En la ESA este porcentaje es muy bajo, lo que la diferencia de otras patologías inflamatorias en que la disminución no es tan pronunciada ${ }^{13,14}$. El poder determinar distintas glicoformas de isoferritinas puede ser aun de más ayuda en la diferenciación de la ESA de otras enfermedades inflamatorias o neoplásicas (incluido linfomas), ya que se ha detectado presencia de glicoformas básicas en etapas activas y precoces de la enfer$\operatorname{medad}^{14}$. Se considera que valores sobre 1.000 $\mathrm{ng} / \mathrm{ml}$ son muy sugerentes de ESA, sin que niveles más bajos o normales constituyan un criterio de exclusión. Más de la mitad de nuestros pacientes tuvo valores de ferritina sobre $1.000 \mathrm{ng} / \mathrm{ml} \mathrm{e}$ incluso 5\% superó los $15.000 \mathrm{ng} / \mathrm{ml}$, lo que fue importante para definir el diagnóstico y posterior evolución. Los niveles de ferritina tendrían importancia pronóstico-terapéutica y enfermos con valores extremadamente altos (>10.000 ng/ml) al momento del diagnóstico, necesitarían desde el inicio tratamiento corticoesteroidal enérgico ${ }^{16}$.

Se postula que la frecuente alteración de las pruebas de función hepática en la ESA (75\% en nuestra serie), en especial alza de transaminasas o fosfatasas alcalinas, derivan de infiltración mononuclear periportal e hiperplasia de las células de Kupffer, demostrado en biopsias hepáticas 9 . 
La serología inmunológica es importante por su negatividad, excluyendo enfermedades autoinmunes, que por sus características son difíciles de diferenciar de una ESA (Tabla 1). Para algunos autores la presencia de FR y de AAN es un factor excluyente, a diferencia de otros, que no los consideran elementos definitorios 5 ,6. En nuestro estudio, un alto porcentaje (94\%) tuvo FR negativo y $90 \%$ AAN negativos. Consideramos que su presencia, como elementos aislados, no deben excluir una ESA, si hay criterios que la apoyen y se han descartado con fundamentos sólidos otras afecciones autoinmunes.

La imagenología no es un aporte significativo precoz; los cambios radiológicos son tardíos e inicialmente inespecíficos, como aumento de partes blandas y osteopenia yuxta articular. En cuadros prolongados puede haber daño articular, que en el carpo y carpometacarpianas puede llegar a la anquilosis, presente en uno de nuestros casos, hallazgo característico de esta enfermedad ${ }^{2,11}$.

El tratamiento ha variado con el tiempo. Clásicamente se usaron altas dosis de AAS (4,0 gramos diarios o más) o AINES, considerándose que una rápida respuesta a estos fármacos constituía, y sigue siendo así, una buena prueba terapéutica $3,8,15$. Sin embargo, las altas dosis necesarias, con los efectos adversos correspondientes, hace difícil su mantención prolongada, además que con frecuencia la respuesta es pobre, o bien al disminuir las dosis tienden a reaparecer los síntomas. No más de $20 \%$ a $25 \%$ de los pacientes responden a esta terapia ${ }^{9}$, siendo sólo $15 \%$ en nuestra experiencia. Actualmente los corticoesteroides sistémicos son de primera elección, en dosis entre 0,5 a $1 \mathrm{mg} / \mathrm{Kg} /$ día de prednisona. Si bien un alto porcentaje responde a esta terapia, en más de un tercio de nuestros casos fue necesario agregar MTX y, en situaciones individuales, azatioprina, sulfazalasina, hidroxicloroquina o leflu- nomida. El uso de pulsos de metilprednisolona es necesario en casos de enfermedad aguda, severa y con riesgo vital ${ }^{3,5,14-19}$. Se han ensayado también otros medicamentos, especialmente en casos recidivantes o de curso persistente, como penicilamina, ciclosporina $\mathrm{A}^{3,9}$ y leflunomida ${ }^{20}$, usada recientemente en una de nuestras pacientes. Últimamente se reporta el uso de agentes biológicos (anti TNFa), como infliximab ${ }^{21-25}$ y etanercept $^{22,26}$, con respuesta variable.

El pronóstico depende del diagnóstico precoz y tratamiento adecuado, que conducen a una evolución favorable preferentemente monocíclica, siendo menos frecuente la recurrente o persistente. Se describen ocasionalmente cuadros severos, incluso catastróficos, acompañados de complicaciones vitales ${ }^{2,3}$. Se han comunicado casos con características de síndrome de respuesta inflamatoria sistémica grave, con shock, síndrome de distrés respiratorio agudo y coagulación intravascular diseminada, por lo que algunos autores consideran que debe ser incluida entre las causas de shock con falla multiorgánica ${ }^{27}$.

En conclusión, la ESA, descrita hace 40 años, no difiere en Chile de la de otros lugares, continúa siendo un problema diagnóstico-terapéutico y especialmente, una gran simuladora. La presencia de fiebre en todos los pacientes, sin existir manifestaciones clínicas ni de laboratorio patognomónicos de alguna enfermedad en especial, constituye un fundamento para ser incluida en el estudio de pacientes con un síndrome febril de origen indeterminado. La sospecha diagnóstica, el descarte de cuadros infecciosos, neoplásicos e inmunológicos junto a exámenes, si bien no específicos, altamente sugerentes, como el alza significativa de la ferritina sérica, permiten establecer su presencia. El diagnóstico precoz y oportuno más un tratamiento adecuado, conducen una evolución favorable en un alto porcentaje de pacientes. 


\section{REFERENCIAS}

1. Bywaters EG. Still's disease in the adult. Ann Rheum Dis 1971; 30: 121-33.

2. Reginato M. Enfermedad de Still del adulto. En: Arís H, Valenzuela F. (eds): Reumatología. $1^{\circ}$ ed. Santiago de Chile. Fundación de Investigación y Perfeccionamiento Médico 1995; pp 161-4.

3. Esdaile JM. Adult Still's Disease. En: Klippel JH, Crofford LJ, Stone JH, Weyand CM, (Eds): PRIMER on the Rheumatic Diseases. $12^{\circ}$ ed. Atlanta, Georgia. Arthritis Foundation 2001; 426-30.

4. Reginato A. Still's Disease, TRAPS, and other episodic febrile syndromes. Reumatología 2003; 19: 100-2.

5. Masson C, Le loet X, Liote F, Dubost JJ, Boisier M-C, Perroux-Goumy L ET al. Comparative study of 6 types of criteria in adult Still's disease. J Rheumatol 1996; 23: 495-97.

6. Chanqueo L. Enfermedad de Still del Adulto y Valor Diagnóstico de la Ferritina Sérica. Reumatología 2001; 17: 179-83.

7. Cush JJ, Medsjer Jr TA, Christy WC, Herbert DC, Cooperstein LA. Adult-onset Still's disease. Clinical course and outcome. Arthritis Rheum 1987; 30: 18694.

8. Yamaguchi M, Оhta A, Tsunematsu T. Preliminary criteria for classification adult Still's disease. J Rheumatol 1992; 19: 424-30.

9. García E, SaAvedra MA. Enfermedad de Still del adulto. Descripción clínico-terapéutica. Rev Méx Reumat 2004; 19: 345-54.

10. Sampalis JS, Medsger Jr TA, Fries JF, Yeadon C, Senecal JL, Myhal D ET aL. Risk Factors for adult Still's Disease. J Rheumatol 1996; 23: 1049-54.

11. EsDaile JM. Adult Still's Disease. En: Klippel JH, Dieppe PA, (eds): Rheumatology. $2^{\mathrm{a}}$ ed. London. Mosby Internacional, 1998.

12. Soto L, Astorga E, Gómez-Veroíza X, Sabugo F, Villanueva M. Linfoadenopatía en Enfermedad de Still del Adulto. Reporte de 4 casos. Reumatología 2004; 20: 215.

13. Akritidis N, Giannakakis Y, Sakkas L. Very High Serum Ferritin Levels in Adult-Onset Still's Disease. Br J Rheumatol 1997; 36: 608-9. (Correspondence).

14. Van Reeth C, Le Moel G, Lasne Y, Revenant MC, Agneray J, Kahn MF, Bourgeols P. Serum Ferritin and isoferritins are tools for diagnosis of active adult Still's Disease. J Rheumatol 1994; 21: 890-5.
15. Vignes S, Le Moël G, Fautrel B, Wechsler B, Godeau P, PIETTE JC. Percentage of glycosylated serum ferritin remains low throughout the course of adult onset Still's disease. Ann Rheum Dis 2000; 59: 347-50.

16. Akritidis N, Giannakakis I, Giouglis T. Ferritin levels and response to treatment in patients with adult Still's disease. J Rheumatol 1996; 23: 201-2.

17. Iglesias J, Sathiraju S, Marik PE. Severe systemic inflammatory response syndrome with shock and ARDS resulting of Still's disease. Clinical response with high-doses pulse methylprednisolone therapy. Chest 1999; 115: 1738-4017.

18. Pouchot J, Vinceneux P. Diagnosis, progresion and prognosis, aetiology and treatment of adult-onset Still's disease. Presse Med 2004; 33: 1019-26.

19. Fujj T, Akizuki M, Kameda H, Matsumura M, Hirakata M, YoshidA T ET AL. Methrotrexate treatment in patients with adult onset Still's disease - retrospective study of 13 Japanese cases. Ann Rheum Dis 1997; 56: 144-8.

20. Cefle A. Leflunomide and azathioprine combination in refractory adult-onset Still's disease. Ann Pharmacother 2005; 39: 764-7.

21. Kraetsch HG, Antoni C, Kalden JR, Manger B. Successful treatment of a small cohort of patients with adult onset of Still's disease with infliximab: first experiences. Ann Rheum Dis 2001; 60: 55-7.

22. Dechant C, Schauenberg P, Antoni CE, Kraetsch HG, Kalden JR, Manger B. [Longterm outcome of TNF blockade in adult-onset Still's disease.] Dtsch Med Wochenschr 2004; 129: 1308-12.

23. Fautrel B, Sibila J, Mariette X, Combe B. Tumour necrosis factor alpha blocking agents in refractory adult Still's disease: an observational study of 20 cases. Ann Rheum Dis 2005; 64: 262-6.

24. Kokkinos A, Oliopoulos A, Greka P, Efthymiou A, Katsilambros N, SfiKakis PP. Successful treatment of refractory adult-onset Still's disease with infliximab. A prospective, non-comparative series of four patients. Clin Rheumatol 2004; 23: 45-9.

25 Bonilla MG, Cobo T, de Miguel E, Martin-Mola E. [Infliximab (anti TNF-alpha) treatment in patients with adult Still's disease. Experience in 2 cases.] An Med Interna 2004; 21: 23-6

26. Husni me, Maier Al, Mease PJ, Overman SS, Fraser P, Gravallese EM, Weinblatt ME. Etanercept in the Treatment of adult Patients with Still's Disease. Arthritis Rheum 2002; 45: 1171-6.

27. Ortega J, Ceres F. Enfermedad de Still del adulto y síndrome de respuesta inflamatoria sistémica. Papel de la ferritina sérica. Medicina Intensiva 2003; 27: 624-6. 